\title{
Correction to: Effect of renal transplantation on cognitive function in hemodialysis patients: a longitudinal study
}

\author{
Yogesh K. Chhabra ${ }^{1} \cdot$ Sanjay Sood ${ }^{2} \cdot$ Omprakash Rathi $^{3} \cdot$ Sandeep Mahajan ${ }^{1}$
}

Published online: 8 December 2017

(c) Springer Science+Business Media B.V., part of Springer Nature 2017

Correction to: Int Urol Nephrol (2017) 49:2071-2078 https://doi.org/10.1007/s11255-017-1700-1

In the original publication, the second author's affiliation was incorrectly published as "Department of Physiology, RAK College of Medical Sciences, Ras Al Khaimah, UAE". The correct affiliation should read as "Visiting Academic, Basic Medical Sciences Department, College of Medicine, University of Sharjah (UAE)".

The original article can be found online at https://doi. org/10.1007/s11255-017-1700-1.

Sandeep Mahajan

mahajansn@yahoo.com

1 Department of Nephrology, All India Institute of Medical Sciences, New Delhi 110029, India

2 Visiting Academic, Basic Medical Sciences Department, College of Medicine, University of Sharjah, Sharjah, UAE

3 Department of Nephrology, Bombay Hospital, Indore 452010, India 